\title{
Single top quark observation at the Tevatron
}

\author{
Sandra Leone ${ }^{* i}$ \\ INFN Sezione di Pisa (Italy) \\ E-mail: sandra.leone@pi.infn.it
}

\begin{abstract}
We report the first observation of single top production in $p \bar{p}$ collisions made by the CDF and D0 collaborations at the Tevatron Collider and the measurement of the inclusive single top cross section in the $s$ and $t$ channels. Both collaborations measured a single top production cross section consistent with the standard model prediction, with a $5.0 \sigma$ significance, and performed the first direct measurement of the CKM matrix element $\left|V_{t b}\right|$. A Bayesian analysis is used to combine $\mathrm{CDF}$ and D0 results and to extract the cross section from the distributions of multivariate discriminants provided by the two collaborations. For a top mass of $170 \mathrm{GeV} / c^{2}$, a Tevatron combined single top cross section of $2.76_{-0.47}^{+0.58}$ is measured. A first attempt to measure the $s$ and $t$ channels cross sections separately is described. The single top candidates sample is used for the first time to measure top properties.
\end{abstract}

XVIII International Workshop on Deep-Inelastic Scattering and Related Subjects, DIS 2010 April 19-23, 2010

Firenze, Italy

\footnotetext{
${ }^{*}$ Speaker.

${ }^{\dagger}$ on behalf of the CDF and DO Collaborations.
} 


\section{Introduction}

In the standard model (SM), top quarks are expected to be produced singly in $p \bar{p}$ collisions through $s$-channel or $t$-channel exchange of a virtual $\mathrm{W}$ boson [1]. The single top production cross section is directly proportional to the square of the CKM [2] matrix element $\left|V_{t b}\right|$. Therefore, a measurement of the single top rate allows a direct measurement of $\left|V_{t b}\right|$ and constraints several new phenomena [3]. Furthermore, understanding single top production represents a milestone towards very challenging searches as the Higgs boson search.

Electroweak production of single top quarks is a rare process at the Tevatron. The combined $s$-channel and $t$-channel cross section is expected to be smaller than those of the background processes, and even smaller than the uncertainty on the total background rate. To overcome these challenges, both CDF and D0 collaborations used a variety of multivariate techniques, for separating single top events from the backgrounds [4].

The analysis described here used data collected by CDF II $\left(3.2 \mathrm{fb}^{-1}\right)$ and D0 $\left(2.3 \mathrm{fb}^{-1}\right)$ detectors. The description of the experimental apparatus can be found elsewhere [5].

\section{Event selection and backgrounds}

Top quarks are expected to decay most of the times to a $W$ boson and a $b$ quark. Both collaborations select events in which a $W$ boson candidate decays leptonically to either an electron or a muon. The presence of at least 2 energetic jets is also required. At least one of the jets has to be identified as containing a $B$-hadron. The background has contributions from events in which a $W$ boson is produced in association with one or more heavy flavor jets, events with mistakenly $b$-tagged light-flavor jets, multijet events (QCD), $t \bar{t}$, diboson events and $Z+$ jets.

In addition CDF performed an analysis selecting events with missing transverse energy and jets (MJ), while vetoing events selected by the lepton + jets analyses, therefore accepting events in which the $W$ boson decays into a tau lepton (which is not reconstructed) or the electron and muon fail the standard lepton identification criteria [6]. To reduce the huge background due to QCD events in this channel, a neural network is used.

D0 recently presented a search for single top quark production using reconstructed tau leptons in the final state, based on $4.8 \mathrm{fb}^{-1}$ [7] (see Figure 1 (right)).

Several multivariate techniques have been used by CDF and D0 [8] [4]. They are: Likelihood Functions (LF and LFS, the second one specific for the $s$-channel), Neural Network (traditional $\mathrm{NN}$ and Bayesian, BNN), Matrix Element (ME), Boosted Decision Trees (BDT). In the CDF MJ analysis the final discriminant is a NN. In the D0 tau analysis the final discriminant is a BDT. Because the multivariate analyses depend heavily on properly simulating events, the Monte Carlo model was checked by comparing hundreds of data and Monte Carlo distributions.

\subsection{Cross section measurement}

The single top cross section is measured using a Bayesian binned likelihood technique assuming a flat prior cross section and integrating over the systematic uncertainties. The measured cross section is the position of the peak of the posterior density distribution and the interval containing $68 \%$ of the integral of the posterior is used to set the \pm 1 sigma uncertainty. 

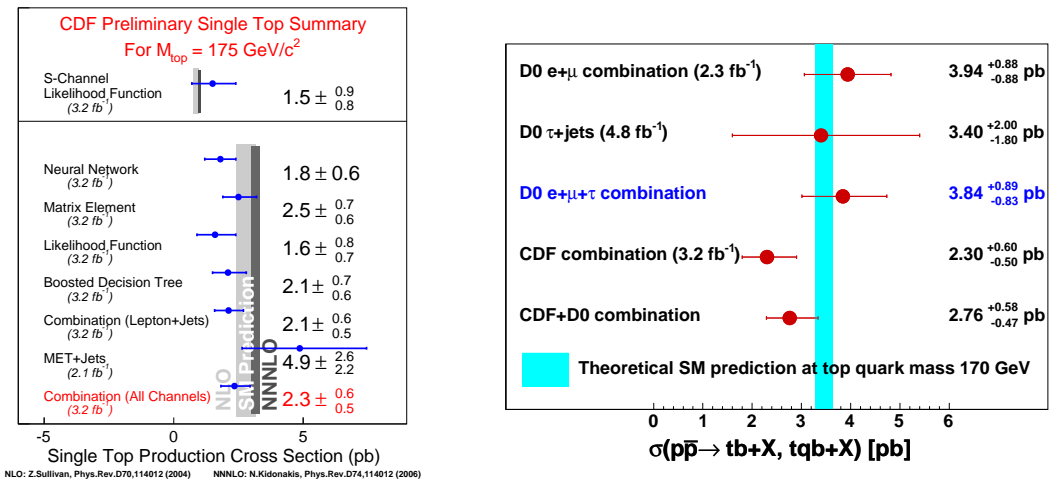

Figure 1: Left: CDF single top various measurements. Right: D0 and CDF cross section measurements and their combination.

Figure 1 (left) shows the CDF single top cross section measurements. The various multivariate methods give consistent results.

D0 combines the ME, BNN and BDT analyses using these discriminants as inputs to a Bayesian neural network, obtaining a single discriminant output. CDF combines the LF, LFS, ME, NN, and BDT results using a super-discriminat (SD) technique. The SD uses a neural network trained with neuro-evolution. A fit over the two exclusive channels ( $\mathrm{SD}$ and $\mathrm{MJ}$ ) is then performed to obtain the final combined result. Figure 1 (right) shows the combined CDF and D0 single top cross section.

\section{3. $V_{t b}$ direct measurement}

The CKM matrix element $\left|V_{t b}\right|^{2}$ is proportional to the single top cross section. In order to extract information about $\left|V_{t b}\right|$ from the cross section measurement we assume that the off-diagonal elements $\left|V_{t s}\right|$ and $\left|V_{t d}\right|$ are much smaller than $\left|V_{t b}\right|$, but we do not make assumptions about the unitarity of the 3 x 3 CKM matrix, allowing for a fourth generation of quarks. We obtain the $\left|V_{t b}\right|^{2}$ posterior by dividing the measured cross section by the theoretical single top cross section. CDF finds $\left|V_{t b}\right|=0.91 \pm 0.11$ (stat.+syst.) \pm 0.07 (theory) and a limit $\left|V_{t b}\right|>0.71$ at $95 \%$ C.L, for a top mass of $175 \mathrm{GeV} / c^{2}$ [9]. D0 finds $\left|V_{t b}\right|=1.07 \pm 0.12$ and a limit $\left|V_{t b}\right|>0.78$ at $95 \%$ C.L, for a top mass of $170 \mathrm{GeV} / c^{2}$ [10]. A flat prior in $\left|V_{t b}\right|^{2}$ from 0 to 1 is assumed for the $95 \%$ C.L. limit results.

\section{CDF and D0 combination}

A Bayesian statistical analysis is performed to combine CDF and D0 single top cross section measurements [11]. Rate and shape uncertainties are categorized by their source and correlations between signals and backgrounds within a channel, between channels and between experiments are evaluated. Sources of systematic uncertainty that are common to the two collaborations are assigned a $100 \%$ correlation. The combination procedure includes the CDF super-discriminant, the CDF missing $E_{T}$ plus jets analysis and the D0 combination discriminant. The combined cross section has been evaluated assuming a top mass of $170 \mathrm{GeV} / \mathrm{c}^{2}$. Figure 3 (left) shows the posterior 

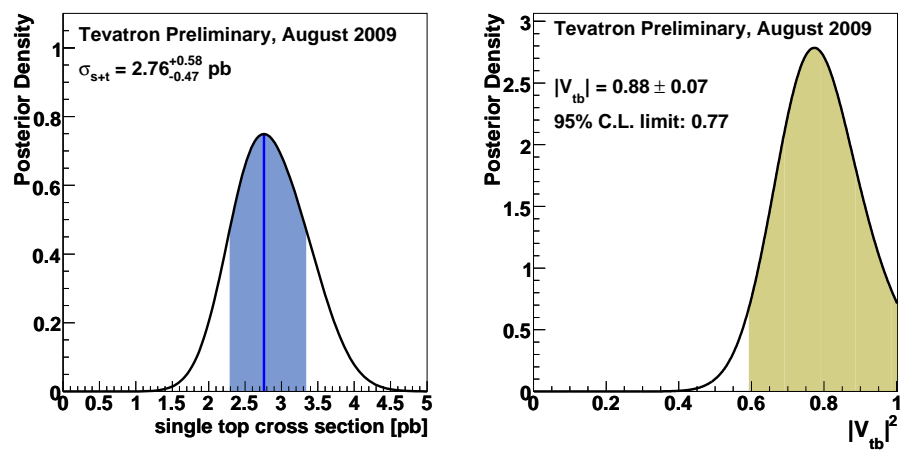

Figure 2: Cross section (left) and $\left|V_{t b}\right|$ (right) Posterior Probability Distribution for the CDF+D0 combined analyses.

\begin{tabular}{lc}
\hline Measured cross section: & $2.76_{-0.47}^{+0.58} \mathrm{pb}$ \\
\hline$\left|V_{t b}\right|, \sigma_{s+t}^{\text {theory }}=3.46 \mathrm{pb}$ & $0.88 \pm 0.07$ \\
$\left|V_{t b}\right| 95 \%$ C.L. limit & 0.77 \\
\hline$\left|V_{t b}\right|, \sigma_{s+t}^{\text {theory }}=3.14 \mathrm{pb}$ & $0.91 \pm 0.08$ \\
$\left|V_{t b}\right| 95 \%$ C.L. limit & 0.79 \\
\hline
\end{tabular}

Table 1: Combined CDF and D0 single top cross section measurement and $\left|V_{t b}\right|$ value and lower limit for two different theory cross sections [9] [10].

probability distribution of the combined CDF and D0 analyses. The combination improves the cross section uncertainty from about $22 \%$ for each experiment to $19 \%$.

A measurement of $\left|V_{t b}\right|$ is obtained from the combined cross section. The relative $\left|V_{t b}\right|$ uncertainty improves from $14 \%$ (CDF) and $11 \%$ (D0) to $8 \%$ for the combination, more than for the cross section due to the square root dependence. Figure 3 (right) shows the posterior probability distribution for $\left|V_{t b}\right|$. Table 1 lists the combined cross section and $\left|V_{t b}\right|$ value.

\section{Separate $t$ and $s$ channel measurements}

Both experiments measured separately the cross section in the $s$ and $t$ channels. CDF interpreted the result of the lepton plus jets and missing $E_{T}$ plus jets analyses in the $\left(\sigma_{s}, \sigma_{t}\right)$ plane (Figure 4 (left)) [8]. The best fit corresponds to: $\sigma_{s}=1.8_{-0.5}^{+0.7} \mathrm{pb}$ and $\sigma_{t}=0.8 \pm 0.4 \mathrm{pb}$. D0 optimized the NN, BDT and ME for the $t$-channel and measured the $s$ and $t$ channels cross sections simultaneously (Figure 4 (right)) obtaining: $\sigma_{s}=1.05 \pm 0.81 \mathrm{pb}$ and $\sigma_{t}=3.14{ }_{-0.80}^{+0.94} \mathrm{pb}(4.8$ standard deviations significance) [12].

\section{First preliminary measurements of top properties in the single top sample}

D0 extracted the total width of the top quark $\Gamma_{t}$. Taking $\Gamma(t \rightarrow W b)$ from the measured $t$ channel cross section and using the measured value of $R=B(t \rightarrow W b) / B(t \rightarrow W q)$ [13], they 

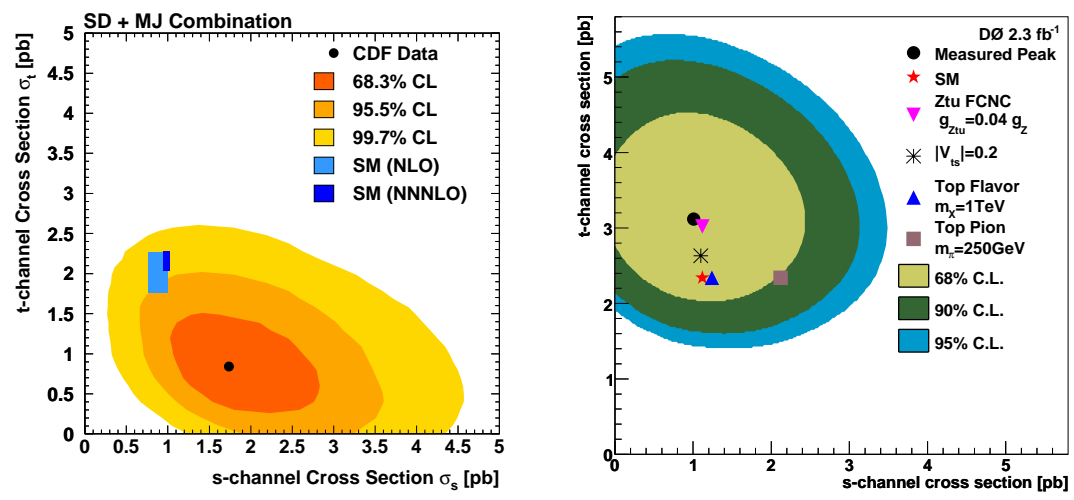

Figure 3: Posterior probability density for $t$-channel and $s$-channel single top quark production in contours of equal probability density for CDF (left) and D0 (right). The SM predictions are also indicated with their theoretical uncertainties.

obtained: $\Gamma_{t}=2.1 \pm 0.6 \mathrm{GeV}$, which translates into a top quark lifetime of $\tau_{t}=(3 \pm 1) \times 10^{-25}$ $s$ [14].

CDF measured the single top polarization and found $\left(\sigma_{R}-\sigma_{L}\right) /\left(\sigma_{R}+\sigma_{L}\right)=-1.0_{-0}^{+1.5}$, consistent with the SM expectation of $100 \%$ left-handed polarization [15].

\section{Conclusions}

CDF and D0 have developed several multivariate analysis techniques to distinguish single top events from the overwhelming background. They established in 2009 the presence of single top events in their data. CDF and D0 cross sections were combined giving the first Tevatron mesurement of single top production and the first direct measurement of the CKM matrix element $\left|V_{t b}\right|$. This achievement was one of the milestones for Run II of the Tevatron. Moreover, it represents an important, necessary benchmark in the search for the Higgs boson at the Tevatron.

\section{References}

[1] S.S.D. Willenbrock and D.A. Dicus, Phys. Rev. D 34, 155 (1986);

[2] N. Cabibbo, Phys. Rev. Lett. 10, 531 (1963); M. Kobayashi and T. Maskawa, Prog. Theor. Phys. 49, 452 (1973).

[3] N.T.M.P. Tait and C.P. Yuan, Phys. Rev. D 66, 054024 (2002).

[4] V. M. Abazov et al., Phys. Rev. Lett. 103, 092001 (2009); T. Aaltonen et al., Phys. Rev. Lett. 103, 092002, (2009).

[5] A. Abulencia et al., J. Phys. G: Nucl. Part. Phys. 34, 2457 (2007); V.M. Abazov et al., Nucl. Instrum. Methods Phys. Res. A 565, 463 (2006).

[6] T. Aaltonen et al., Phys. Rev. D 81, 072003 (2010).

[7] V. M. Abazov et al., Phys. Lett. B 690, 5 (2010). 
[8] T. Aaltonen et al., Phys. Rev. D 81, 072003 (2010).

[9] B.W. Harris et al., Phys. Rev. D 66, 054024 (2002).

[10] N. Kidonakis, Phys. Rev. D 74, 114012 (2006).

[11] Tevatron Electroweak Working Group for the CDF and D0 Collaborations. arXiv:0903.2503, (2009).

[12] V. M. Abazov et al., Phys. Lett. B 682, 363 (2010).

[13] V. M. Abazov et al., Phys. Rev. Lett. 100, 192003 (2008).

[14] D0 CONF-note 6034.

[15] CDF CONF-note 9920. 